Discrete Comput Geom 27:137-154 (2002)

DOI: $10.1007 / \mathrm{s} 00454-001-0057-4$

\title{
Examples of $\mathbb{Z}$-Acyclic and Contractible Vertex-Homogeneous Simplicial Complexes*
}

\author{
F. H. Lutz
}

Fakultät II - Mathematik und Naturwissenschaften,

Institut für Mathematik, Sekr. MA 6-2, Technische Universität Berlin,

Straße des 17. Juni 136, D-10623 Berlin, Germany

lutz@math.tu-berlin.de

\begin{abstract}
It was shown in [11] that there are no (non-trivial) two- and three-dimensional $\mathbb{Z}$-acyclic vertex-homogeneous simplicial complexes. In this paper we construct a fivedimensional example and further examples in higher dimensions, one of which is Oliver's example of dimension 11 , the only previously known example of a non-contractible $\mathbb{Z}$ acyclic vertex-homogeneous simplicial complex. We also present an infinite series of contractible vertex-homogeneous simplicial complexes by starting with one of the $\mathbb{Z}$-acyclic examples.
\end{abstract}

\section{Introduction}

Interest in vertex-homogeneous simplicial complexes with certain topological properties can arise from different perspectives. For example, such complexes appear naturally when one studies certain fixed point theorems in algebraic topology but they also show up in connection with the famous Evasiveness Conjecture in complexity theory. This astonishing conjunction was established by Kahn et al. in [10] where they made use of a fixed point theorem by Oliver [12] to settle the Evasiveness Conjecture in the prime power case (and for $n=6$ ).

In some sense, at the core of the connection is the observation that if the vertextransitive action of a (finite) group $G$ on a (finite) simplicial complex $K$ (with $m$ vertices) has a fixed point, then $K$ is a simplex. To see this geometrically, we may regard $K$ as a subcomplex of the standard ( $m-1$ )-dimensional simplex $\Delta_{m-1}$ with vertices $e_{1}, \ldots, e_{m}$. Any point $x$ of $K$ then has a unique representation $x=\sum_{i=1}^{m} \lambda_{i} e_{i}$, with $\lambda_{i} \geq 0$ and

\footnotetext{
* This work was supported by the graduate school "Algorithmische Diskrete Mathematik" funded by the Deutsche Forschungsgemeinschaft (DFG), Grant GRK 219/2-97.
} 
$\sum_{i=1}^{m} \lambda_{i}=1$. The group $G$ acts by permuting the coordinates, $g x=\sum_{i=1}^{m} \lambda_{i} e_{g(i)}$, $g \in G$. If $G$ is transitive, then for every $i, j$ there is some $g \in G$ such that $e_{j}=e_{g(i)}$. If, in addition, the action of $G$ has a fixed point $y$, then $g y=y$ for every group element $g$, and therefore $\lambda_{1}=\cdots=\lambda_{m}=1 / m$. However, $y=(1 / m) \sum_{i=1}^{m} e_{i}$ is a point of $K$ if and only if $K$ is the simplex $\Delta_{m-1}$.

For certain group actions the existence of such fixed points can be guaranteed by fixed point theorems. It was shown by Smith [15] that if a $p$-group $P$, i.e., a group with prime power order $|P|=p^{t}$, acts on a $\mathbb{Z}_{p}$-acyclic complex, then the fixed point set for this action is $\mathbb{Z}_{p}$-acyclic as well. In particular, the fixed point set is not empty-hence, there are no vertex-transitive group actions of a $p$-group on a $\mathbb{Z}_{p}$-acyclic simplicial complex (that is not a simplex).

The theorem by Smith has been generalized by Oliver.

Theorem 1 [12]. Let $G$ be a finite group with subsequent normal subgroups $P \triangleleft Q \triangleleft G$ such that

(i) $P$ is a p-group,

(ii) $G / Q$ is a $q$-group, and

(iii) $Q / P$ is cyclic.

If $G$ acts on a $\mathbb{Z}_{p}$-acyclic complex $K$, then the Euler characteristic $\chi\left(K^{G}\right)$ of the fixed point set $K^{G}$ is equivalent to $1(\bmod q)$.

In order to give a brief account on the Evasiveness Conjecture, let $\mathcal{P}$ be any graph property, that is, a property of graphs which is invariant under graph-isomorphisms, on a fixed set of nodes $V$ of size $n:=|V|$, and let $E$ denote the set of all edges on $V$, with $m:=|E|=\left(\begin{array}{l}n \\ 2\end{array}\right)$. We identify $\mathcal{P}$ with the set system

$$
\mathcal{F}_{\mathcal{P}}:=\{A \subseteq E \text { : graph }(V, A) \text { has property } \mathcal{P}\} \subseteq 2^{E},
$$

and for an unknown graph $\mathcal{G}=(V, A)$ on $V$ we consider the decision problem whether $\mathcal{G}$ has the property $\mathcal{P}$ or not. In order to find out if the edge set $A$ of $\mathcal{G}$ belongs to $\mathcal{F}_{\mathcal{P}}$, we ask questions of the type "Is $e \in A$ ?", and an oracle answers (correctly) YES or NO.

The number of elements of $E$ that we will have to test in the worst case, if we proceed according to some optimal strategy, is called the argument complexity $c\left(\mathcal{F}_{\mathcal{P}}\right)$ of $\mathcal{P}$. Then $0 \leq c\left(\mathcal{F}_{\mathcal{P}}\right) \leq m$, and $\mathcal{P}$ is trivial if $c\left(\mathcal{F}_{\mathcal{P}}\right)=0$ and non-trivial if $c\left(\mathcal{F}_{\mathcal{P}}\right)>0$. $\mathcal{P}$ is called evasive if $c\left(\mathcal{F}_{\mathcal{P}}\right)=m$ and non-evasive otherwise. For general set systems $\mathcal{F} \subseteq 2^{E}$, these terms are defined analogously. A graph property is monotone if it is preserved under deletion of edges.

In the early seventies Richard Karp proposed the following remarkable conjecture.

Evasiveness Conjecture for Graph Properties. Every non-trivial monotone graph property $\mathcal{P}$ is evasive.

Extensive work has been done on determining the argument complexity of particular graph properties (see, e.g., [1], [2], [5, Chapter VIII], and [19]).

Kahn et al.'s approach to the Evasiveness Conjecture was by reformulating Karp's conjecture in the language of simplicial complexes: if $\mathcal{P}$ is a monotone graph property, 
then the corresponding set system $\mathcal{F}_{\mathcal{P}}$ is a (finite abstract) simplicial complex with vertex set $E$. We denote by $\mathcal{F}_{\mathcal{P}}$ the graph complex associated with $\mathcal{P}$. Invariance under permutation of the nodes of $V$ (what one naturally requires for $\mathcal{P}$ to be a graph property) gives rise to an induced action of the symmetric group $S_{n}$ on the edge set $E$, and thus on the simplicial complex $\mathcal{F}_{\mathcal{P}}$. Clearly, the action of $S_{n}$ is transitive on $E$.

Theorem 2 [10]. Let $\mathcal{F}_{\mathcal{P}_{n}}$ be the graph complex associated with some (non-trivial) graph property $\mathcal{P}_{n}$ on $n=p^{t}$ nodes, with $p$ prime. Then $\mathcal{F}_{\mathcal{P}_{n}}$ is not $\mathbb{Z}_{p}$-acyclic.

Proof. Let $G=\operatorname{Aff}\left(G F\left(p^{t}\right)\right)<S_{n}$ be the group of affine transformations of $G F\left(p^{t}\right)$ and let $Q:=G$ and $P:=\left\{x \mapsto x+b: b \in G F\left(p^{t}\right)\right\}$. The group $G$ is 2-transitive on $\{1, \ldots, n\}$ and therefore transitive on the edge set $E$. Hence, $G$ is a vertex-transitive subgroup of the symmetric group $S_{n}$ with induced action on all graph complexes $\mathcal{F}_{\mathcal{P}_{n}}$. However, then either $\mathcal{F}_{\mathcal{P}_{n}}$ is a simplex, and thus $\mathcal{P}_{n}$ is trivial, or $\mathcal{F}_{\mathcal{P}_{n}}$ is not $\mathbb{Z}_{p}$-acyclic by Theorem 1 and the particular choice of $Q$ and $P$.

If a graph complex is not $\mathbb{Z}_{p}$-acyclic, then it cannot be non-evasive (see below).

Corollary 3 [10]. The Evasiveness Conjecture for graph properties holds for every prime power number of nodes.

By considering arbitrary vertex-homogeneous simplicial complexes one obtains the following more general situation.

Evasiveness Conjecture for Simplicial Complexes [10]. If $\mathcal{F}$ is a non-evasive vertexhomogeneous simplicial complex on the vertex set $E=\{1, \ldots, m\}$ with vertex-transitive action by some group $G$, then it is the standard $(m-1)$-simplex $\Delta_{m-1}$.

To be "non-evasive" is a strong topological requirement. The following sequence of implications holds for finite simplicial complexes (see [3], [10], and [18]):

non-evasive $\Rightarrow$ collapsible $\Rightarrow$ contractible $\Rightarrow \mathbb{Z}$-acyclic $\Rightarrow \mathbb{Q}$-acyclic $\Rightarrow \tilde{\chi}=0$,

and leads to further generalizations of the above conjecture if we replace "non-evasive" with the respective weaker requirements ( $\tilde{\chi}$ denotes the reduced Euler characteristic of a simplicial complex).

These generalized conjectures all hold once again for prime power numbers of vertices by a theorem of Rivest and Vuillemin [13] (see also [11]). Yet, for non-prime power numbers there are counterexamples known to all of the generalized conjectures with the exception of the Evasiveness Conjecture for simplicial complexes which still remains open.

There is an abundance of (non-trivial) $\mathbb{Q}$-acyclic vertex-homogeneous simplicial complexes and even more with $\tilde{\chi}=0$. The smallest $\mathbb{Q}$-acyclic example is the 6-vertex triangulation of the real projective plane (see below and [11]).

In [11] it was shown that there are no two- and three-dimensional $\mathbb{Z}$-acyclic vertex-homogeneous simplicial complexes other than a simplex. In Section 4 we present 
a five-dimensional example and further examples in higher dimensions, one of which is Oliver's example of dimension 11, the only previously known example of a noncontractible $\mathbb{Z}$-acyclic vertex-homogeneous simplicial complex. In Section 2 we discuss some topological tools that will be used for our later constructions, and in Section 3 we give an infinite series of contractible vertex-homogeneous simplicial complexes. It remains to mention that Oliver described techniques to construct a collapsible vertexhomogeneous simplicial complex (see [10]). On the other hand, evasiveness is preserved by various standard constructions as, for example, join or barycentric subdivision [18]. Thus, in conclusion, the present techniques seem to be insufficient to settle the Evasiveness Conjecture for simplicial complexes or to construct better counterexamples than the $\mathbb{Z}$-acyclic and contractible ones discussed in this paper, which leaves Karp's original Evasiveness Conjecture for graph properties wide open.

\section{Topological Tools}

The main tools for our constructions are the well-known nerve operation and duality observations.

\subsection{The Nerve Operation}

Let $K$ be a (finite abstract) simplicial complex, let $\mathcal{F}=\left(F_{j}\right)_{j \in J}$ be its collection of maximal faces (facets), and let $J$ be the corresponding index set. We call the covering of $K$ by its facets $\mathcal{F}$ the standard covering of $K$.

Theorem 4 (Nerve Theorem, Borsuk, see [3]). Let $\mathcal{N}(K)$ be the nerve complex of $K$ (with respect to the standard covering $\left.\mathcal{F}=\left(F_{j}\right)_{j \in J}\right)$, that is, $\mathcal{N}(K)$ is the simplicial complex on the vertex set $J$ such that $\Delta \subseteq J$ is a simplex of $\mathcal{N}(K)$ if and only if $\bigcap_{j \in \Delta} F_{j} \neq \emptyset$. Then $K$ and $N:=\mathcal{N}(K)$ are homotopy equivalent.

The nerve complex of a simplex of any dimension is a point, and Grünbaum describes in [8] the class of all simplicial complexes having the same nerve. Moreover, by a theorem of Mani (see [8]), there is for every simplicial complex $N$ some simplicial complex $K$ such that $N=\mathcal{N}(K)$.

Definition 5. Let $E$ be the vertex set of $K$, and for every vertex $e$ of $K$ let $e^{1}, \ldots, e^{n}$ be $n$ distinct copies. The $\boldsymbol{n}$ th multiple $\boldsymbol{n} K$ of $K$ is the simplicial complex on the vertex set $n E=\bigcup_{r=1}^{n} E^{r}$, where $E^{r}$ denotes the $r$ th copy of $E$, that has as its maximal faces the sets $n F=\left\{e_{1}^{1}, e_{1}^{2}, \ldots, e_{1}^{n}, \ldots, e_{k}^{1}, e_{k}^{2}, \ldots, e_{k}^{n}\right\}$ for the facets $F=\left\{e_{1}, \ldots, e_{k}\right\}$ of $K$.

By construction, $\bigcap_{j \in \Delta} F_{j} \neq \emptyset$ if and only if $\bigcap_{j \in \Delta} n F_{j} \neq \emptyset$. Hence, $\mathcal{N}(n K)=\mathcal{N}(K)$, and $n K$ is homotopy equivalent to $K$.

Although the above examples demonstrate that there are always combinatorially nonisomorphic simplicial complexes which have the same nerve complex, the nerve operation is injective on a large class of simplicial complexes. Grünbaum [8] calls a simplicial complex taut if every vertex is the intersection of the facets containing it. 
Lemma 6 (Duality [8]). If $K$ is taut, then $\mathcal{N}(K)$ is taut and $K=\mathcal{N}(\mathcal{N}(K))$.

Proof. Let $K$ be taut with standard covering $\mathcal{F}=\left(F_{j}\right)_{j \in J}$. The nerve $N=\mathcal{N}(K)$ has one vertex $j$ for every facet $F_{j}$ of $K, j \in J$. If $\Delta_{e} \subseteq J$ is the collection of all $j$ 's so that the corresponding facets $F_{j}$ contain the vertex $e \in E$, then, by the tautness of $K$, $\bigcap_{j \in \Delta_{e}} F_{j}=\{e\}$ and $\Delta_{e}$ is a facet of $N$. Hence, to any vertex $e$ of $K$ there uniquely corresponds a facet $\Delta_{e}=\left\{j: e \in F_{j}\right\}$ of $N$.

On the other hand, let $j \in J$. Suppose there exists some $j^{\prime} \in J, j^{\prime} \neq j$, such that $j^{\prime} \in \bigcap_{e \in E, j \in \Delta_{e}} \Delta_{e}$. However, then $F_{j} \subset F_{j^{\prime}}$, which is a contradiction to the maximality of $F_{j}$. Thus, the nerve $N$ is taut, and $\mathcal{N}(\mathcal{N}(K))=K$.

The nerve complex $\mathcal{N}(K)$ of a simplicial complex $K$ need not be taut. For example, the nerve complex of a path of length $n$ is a path of length $(n-1)$. A point is taut, and $n$-gons $C_{n}$ are taut for $n \geq 3$.

\subsection{The Join and the Dual Join Product}

Recall that the join $K * K^{\prime}$ of two simplicial complexes $K$ and $K^{\prime}$ (with disjoint vertex sets) is defined as $K * K^{\prime}:=\left\{\Delta \cup \Delta^{\prime}: \Delta \in K, \Delta^{\prime} \in K^{\prime}\right\}$.

Lemma 7. If $K$ and $K^{\prime}$ are taut simplicial complexes, different from a point, then their join product $K * K^{\prime}$ is taut as well.

Proof. The vertex set of $K * K^{\prime}$ is $E \cup E^{\prime}$. Let $e$ be a vertex of $K * K^{\prime}$ with $e \in E$. If $\mathcal{V}=\left(F_{j} \cup F_{j^{\prime}}^{\prime}\right)_{\left(j \in J, j^{\prime} \in J^{\prime}\right)}$ denotes the collection of maximal faces of $K * K^{\prime}$ for the facets $\mathcal{F}=\left(F_{j}\right)_{j \in J}$ of $K$ and the facets $\mathcal{F}^{\prime}=\left(F_{j^{\prime}}^{\prime}\right)_{j^{\prime} \in J^{\prime}}$ of $K^{\prime}$, then

$$
\bigcap_{\substack{j \in J, j^{\prime} \in J^{\prime}, e \in F_{j} \cup F_{j^{\prime}}}} F_{j} \cup F_{j^{\prime}}^{\prime}=\bigcap_{\substack{j \in J, e \in F_{j}}} F_{j}=\{e\},
$$

and the same is true for $e \in E^{\prime}$. Therefore, $K * K^{\prime}$ is taut.

Definition 8. Let $K$ and $K^{\prime}$ be (finite abstract) simplicial complexes. Then the dual join product of $K$ and $K^{\prime}$ is the product

$$
K \bowtie K^{\prime}:=\mathcal{N}\left(\mathcal{N}(K) * \mathcal{N}\left(K^{\prime}\right)\right)
$$

The dual join product of two (non-trivial) taut complexes $K$ and $K^{\prime}$ is taut by Lemmas 6 and 7. In particular, the following equality holds for (non-trivial) taut complexes:

$$
\mathcal{N}\left(K \bowtie K^{\prime}\right)=\mathcal{N}(K) * \mathcal{N}\left(K^{\prime}\right) .
$$




\section{Vertex-Homogeneous Simplicial Complexes}

\subsection{The Nerve of a Vertex-Homogeneous Simplicial Complex}

Let $G$ be a permutation group of the vertex set $E$, and let $K \subseteq 2^{E}$ be a simplicial complex which is invariant under the given $G$-action.

Lemma 9. The action of $G$ on $K$ induces an action of $G$ on the nerve $\mathcal{N}(K)$ of $K$.

Proof. Let $\mathcal{F}=\left(F_{j}\right)_{j \in J}$ be the standard covering of $K$. The action of $G$ on the set $J$ of facets of $K$ gives rise to an action of $G$ on the nerve $\mathcal{N}(K)$, since $\bigcap_{j \in \Delta} g F_{j} \neq \emptyset$ if and only if $\bigcap_{j \in \Delta} F_{j} \neq \emptyset$ for any $\Delta \in \mathcal{N}(K)$.

Let, from now on, $K$ be a vertex-homogeneous simplicial complex. Then the induced action of $G$ on the nerve $N$ of $K$ is, in general, not vertex-homogeneous anymore. More precisely, the action of $G$ on $N$ is transitive on the set of vertices of $N$ if and only if $K$ has exactly one orbit of maximal faces.

Lemma 10 (Characterization of Vertex-Homogeneous Simplicial Complexes).

(i) If $K$ is vertex-homogeneous, then its nerve $N=\mathcal{N}(K)$ is facet-homogeneous.

(ii) If $N$ is facet-homogeneous, then its nerve $K=\mathcal{N}(N)$ is vertex-homogeneous.

Proof. (i) Let $K$ be a vertex-homogeneous simplicial complex on $n$ vertices.

We first show that $N=\mathcal{N}(K)$ is pure. Let $\operatorname{Max}(K)$ denote the collection of orbits of maximal faces of $K$. By transitivity, every vertex $e \in E$ is contained the same number of times, $r_{\mathcal{O}}$, in the $k$-element sets of any particular orbit $\mathcal{O}$ of facets of $K$, i.e., $k \cdot|\mathcal{O}|=r_{\mathcal{O}} \cdot n$. Altogether, every vertex $e$ is contained in precisely $r=\sum_{\mathcal{O} \in \operatorname{Max}(K)} r_{\mathcal{O}}$ distinct facets of $K$, i.e., $\operatorname{dim}\left(\Delta_{e}\right)=r-1$ for every $e$. In particular, $\operatorname{dim}(\mathcal{N}(K))=r-1$.

Let $\Delta$ and $\Delta^{\prime}$ be two different facets of $N$. Then $\Delta$ and $\Delta^{\prime}$ correspond to two distinct sets of $r$ maximal faces of $K$, respectively. Let $e$ be some element in the intersection of the $r$ maximal faces of $K$ corresponding to $\Delta$ (there can be more than one such element if $K$ is not taut!), and let $e^{\prime}$ be an element of $E$ representing $\Delta^{\prime}$. Since the action of $G$ is transitive on $E$, there exists a group element $g \in G$ such that $e^{\prime}=g e$. However, then $g$ maps the facets corresponding to $e$ to the facets corresponding to $e^{\prime}$, and hence the action of $G$ on $N$ is transitive on the facets of $N$.

(ii) Trivial, since any facet-homogeneous simplicial complex has only one orbit of maximal faces.

Example 1. The $n$-gon $C_{n}, n \geq 3$, with rotations by elements of $\mathbb{Z}_{n}$ is a taut vertexhomogeneous and facet-homogeneous simplicial complex with $C_{n}=\mathcal{N}\left(C_{n}\right)$.

If we replace every edge of the circle $C_{n}$ by an $m$-simplex, $m \geq 2$, then the resulting $(n, m)$-necklace $C_{n}^{m}$ is a facet-homogeneous pure simplicial complex with $C_{n}=\mathcal{N}\left(C_{n}^{m}\right)$. Thus, $(n, m)$-necklaces form an infinite class of facet-homogeneous simplicial complexes, which all have the same nerve. 

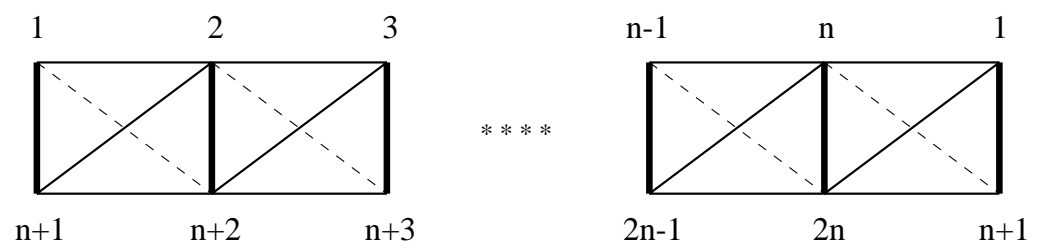

Fig. 1. The two-fold multiple $2 C_{n}$.

Example 2. For $n \geq 3$, the two-fold multiple $2 C_{n}$ is a chain of tetrahedra (Fig. 1): with respect to the action of $\mathbb{Z}_{n} \times \mathbb{Z}_{2}$, which rotates the tetrahedra and flips the upper and lower vertices, the two-fold multiple $2 C_{n}$ is vertex-homogeneous and facet-homogeneous, with $C_{n}=\mathcal{N}\left(2 C_{n}\right)$.

Example 3. The 6-vertex triangulation $\mathbb{R} \mathbf{P}_{6}^{2}$ (see Fig. 2) of the real projective plane is vertex-homogeneous and taut.

The symmetry group of $\mathbb{R} \mathbf{P}_{6}^{2}$ is $A_{5}(6)=\langle(1,2,3,4,6),(1,4)(5,6)\rangle$, with $A_{5}(6)$ acting transitively on the set of maximal faces:

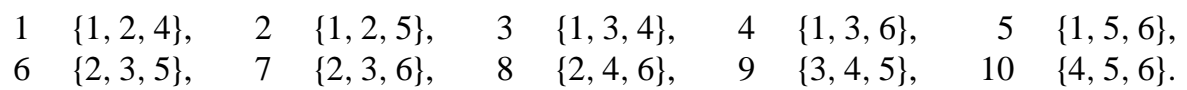

The nerve of $\mathbb{R} \mathbf{P}_{6}^{2}$ is a taut four-dimensional vertex-homogeneous and facet-homogeneous simplicial complex on 10 vertices:

$\begin{array}{llllll}1 & \{1,2,3,4,5\}, & 2 & \{1,2,6,7,8\}, & 3 & \{3,4,6,7,9\}, \\ 4 & \{1,3,8,9,10\}, & 5 & \{2,5,6,9,10\}, & 6 & \{4,5,7,8,10\} .\end{array}$

If we compute the nerve of this complex, then we get $\mathbb{R} \mathbf{P}_{6}^{2}$ again.

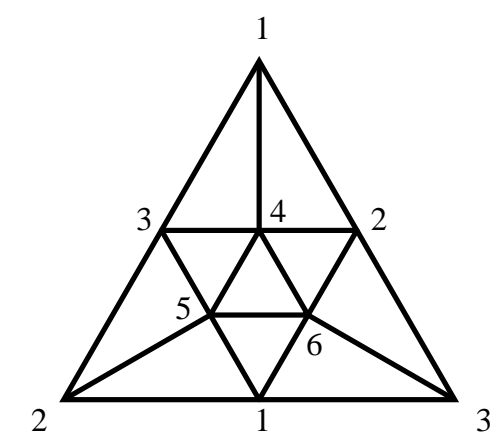

Fig. 2. The 6-vertex triangulation of the real projective plane. 

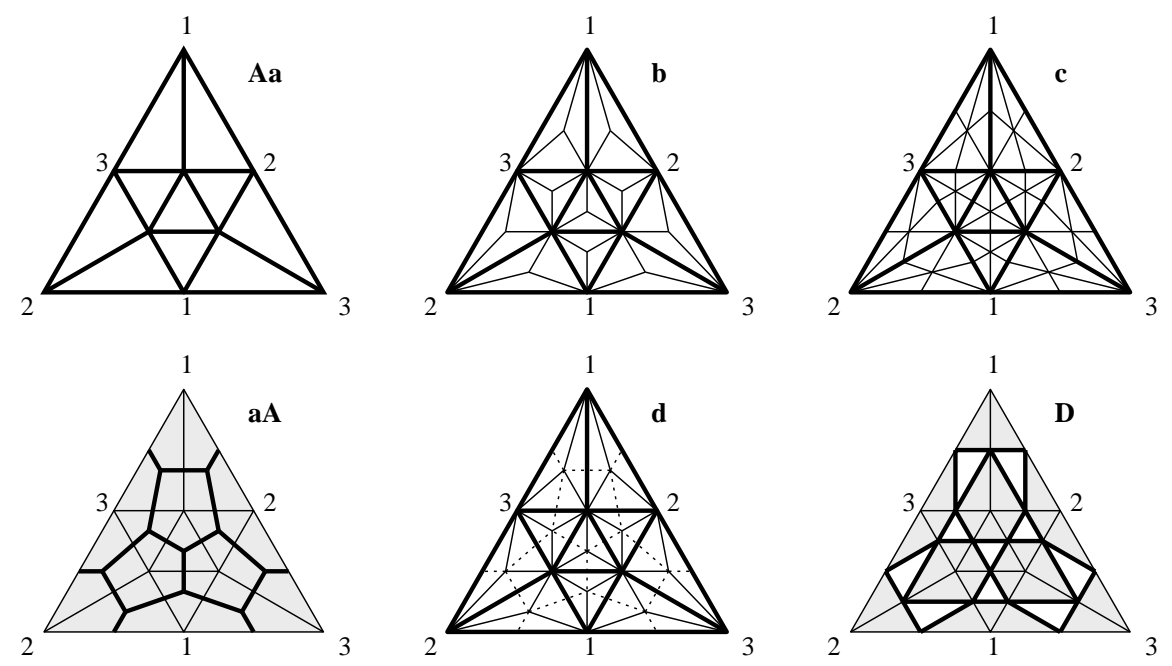

Fig. 3. Vertex/facet-homogeneous simplicial complexes homotopy equivalent to $\mathbb{R} \mathbf{P}^{2}$.

Remark. We see by this example that the nerve of a vertex-homogeneous complex can have more vertices and can be of higher dimension than the original complex, it can also have less vertices and can be of lower dimension.

Example 4 (QQ-Acyclic Vertex-Homogeneous Simplicial Complexes). It turned out in [11] that the 6-vertex triangulation of the real projective plane is the smallest (nontrivial) example of a $\mathbb{Q}$-acyclic vertex-homogeneous simplicial complex. We make use of Lemma 10 to derive further examples of vertex-homogeneous as well as facethomogeneous $\mathbb{Q}$-acyclic simplicial complexes, which are homotopy equivalent to $\mathbb{R} \mathbf{P}^{2}$.

As well as being vertex-homogeneous, the 6-vertex triangulation of the projective plane Aa (see Fig. 3) is facet-homogeneous, as was mentioned above. Hence, the nerve complex aA of Aa (in Fig. 3, vertex-homogeneous and facet-homogeneous simplicial complexes are labeled by capital letters and lowercase letters, respectively) is a facet-homogeneous and vertex-homogeneous simplicial complex on 10 vertices with the shaded pentagons depicting the six four-dimensional facets glued together along the sides of the pentagons.

The nerve complex $\mathbf{B}(\mathbf{C})$ of the facet-homogeneous subdivision $\mathbf{b}$ (of the facet-homogeneous barycentric subdivision $\mathbf{c}$ ) of $\mathbf{A a}$ is vertex-homogeneous on 30 (60) vertices.

If in $\mathbf{b}$ for any bold edge we replace both neighboring triangles by a tetrahedron, then the resulting simplicial complex $\mathbf{d}$ is still facet-homogeneous. Its nerve complex $\mathbf{D}$ is on 15 vertices with two orbits of maximal faces, one consisting of six 4-simplices (shaded pentagons) and the other of 10 triangles. The same construction can be carried through for $\mathbf{c}$ leading to complexes $\mathbf{e}$ and $\mathbf{E}$ (the latter on 30 vertices).

A further example $\mathbf{F}$ of a vertex-homogeneous simplicial complex on 15 vertices, homotopy equivalent to the projective plane, can be obtained from $\mathbf{D}$ by gluing in 60 tetrahedra in the following way. For any white triangle of $\mathbf{D}$ we add six tetrahedra with vertex-sets the triangle and in addition one vertex of a neighboring white triangle, 
respectively. The resulting space $\mathbf{F}$ is still vertex-homogeneous, and it can be worked out easily that it collapses to $\mathbf{D}$ and thus is homotopy equivalent to $\mathbf{D}$.

\subsection{Constructions with Vertex-Homogeneous Simplicial Complexes}

As we have now seen some simple examples of vertex-homogeneous simplicial complexes, we next discuss three constructions that allow us to derive further vertex-homogeneous simplicial complexes if we start with a given one.

Proposition 11. Let $(K, G)$ denote a pair of a simplicial complex $K$ with vertex set $E$ of cardinality $m$ and a group $G<S_{m}$ that acts vertex-transitively on $K$. If $F$ is a finite set with $n=|F|$ elements and $H<S_{n}$ is a transitive permutation group of degree $n$, which acts on $F$, then the simplicial complexes

(i) $\left(K^{* n}, G \times H\right)($ Oliver, see [10]),

(ii) $(K \bowtie F, G \times H)$, and

(iii) $(n K, G \times H)$

are vertex-homogeneous for the obvious actions of $G \times H$.

Proof. (i) Let the direct product $G \times H$ act on the $n$-fold join product $K^{* n}$, with $G$ acting transitively on every copy of $K$ and with $H$ permuting the $n$ copies of $K$. The vertex set of $K^{* n}$ is the union $E_{\cup}=\bigcup_{r=1}^{n} E^{r}$ of $n$ copies of $E$, and the action of $G \times H$ is clearly transitive on $E_{\cup}$.

(ii) Since $(K, G)$ and $(F, H)$ are vertex-homogeneous complexes, their nerve complexes $(\mathcal{N}(K), G)$ and $(\mathcal{N}(F), H)$ are facet-homogeneous, with $\mathcal{N}(F)=F$. The join product $\mathcal{N}(K) * F$ is facet-homogeneous for the diagonal action of $G \times H$, and thus $(K \bowtie F, G \times H)$ is vertex-homogeneous.

(iii) As in (i), $G \times H$ acts transitively on $E_{\cup}=\bigcup_{r=1}^{n} E^{r}$.

Corollary 12. If $K$ is a $\mathbb{Z}$-acyclic vertex-homogeneous simplicial complex, then the $n$-fold multiples $n K$ form an infinite series of $\mathbb{Z}$-acyclic vertex-homogeneous simplicial complexes. Moreover, the series $K^{* n}$ and $K \bowtie F$ provide examples of contractible vertex-homogeneous simplicial complexes.

Proof. It remains to show that $K^{* n}$ and $K \bowtie F$ are contractible for $n \geq 2$. If $K$ is $\mathbb{Z}$-acyclic, then it is connected. Now, the join product of a $k$-connected complex with an $l$-connected complex is $(k+l+2)$-connected. In particular, $K^{* n}$ and $K \bowtie F$ are at least $(0-1+2)$-connected, that is, simply connected. However, since a simplicial complex is contractible if and only if it is simply connected and $\mathbb{Z}$-acyclic (see [3]), the result follows. 


\section{The Identified Dodecahedron and Seven Related $\mathbb{Z}$-Acyclic Vertex-Homogeneous Simplicial Complexes}

We consider the boundary complex of the dodecahedron with 12 pentagonal facets, 30 edges, and 20 vertices. If we identify opposite pentagons by a coherent twist of $\pi / 5$ radians, then the resulting cell complex $Q$ is $\mathbb{Z}$-acyclic; see Fig. 4 and [4], [6, p. 57], and [7]. The symmetry group of the identified dodecahedron $Q$ is the alternating group $A_{5}$. Note that the identified dodecahedron is simply the 2-skeleton of the Poincare homology 3 -sphere in its description by Threlfall and Seifert [16] and Weber and Seifert [17] as the spherical dodecahedron space, which is given by the full dodecahedron with the same identifications on the boundary as above.

Lemma 13. There are precisely two $A_{5}$-invariant facet-transitive triangulations of the $\mathbb{Z}$-acyclic complex $Q$.

Proof. Every edge of the cell complex $Q$ is the intersection of three pentagonal cells, which implies that the 1-skeleton of $Q$ is necessarily included in the 1-skeleton of any $A_{5}$-invariant triangulation of $Q$. This is also the case for the five vertices of $Q$. The action of $A_{5}$ on $Q$ is transitive on the pentagons, and any of the pentagons has the dihedral group $D_{5}<A_{5}$ as its isotropy group. It is therefore sufficient to determine $D_{5}$-invariant facet-transitive triangulations of a pentagon. There are exactly two such triangulations, one with 5 and the other with 10 triangles. We denote the corresponding triangulations of $Q$ with 30 and 60 triangles by $N_{\mathrm{I}}$ and $N_{O}$, respectively (see Figs. 6 and 5).

Corollary 14. The nerve complexes $K_{1}:=\mathcal{N}\left(N_{\mathrm{I}}\right)$ and $K_{O}:=\mathcal{N}\left(N_{O}\right)$ are examples of $\mathbb{Z}$-acyclic but not contractible vertex-homogeneous simplicial complexes on 30 and 60 vertices, respectively.

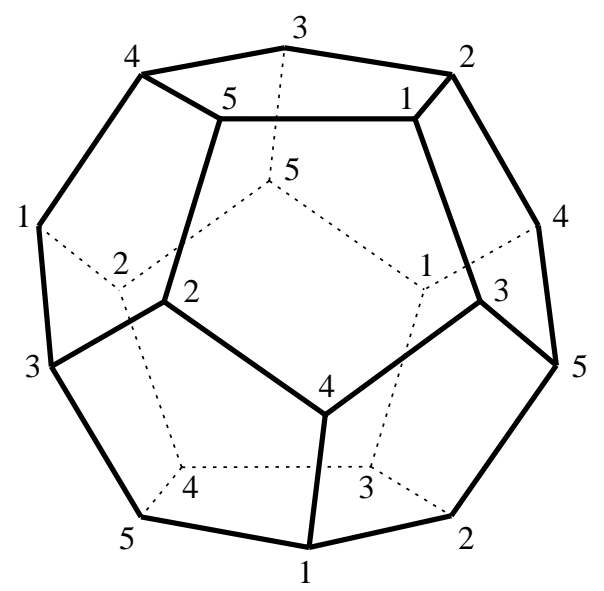

Fig. 4. The $\mathbb{Z}$-acyclic identified dodecahedron. 
Oliver's Example $K_{O}$. The complex $K_{O}$ was first found by Bob Oliver. His construction is purely algebraic and was mentioned in [10] and in a paper by Segev [14]. In fact, Segev presented an explicit proof that the nerve complex $N_{O}=\mathcal{N}\left(K_{O}\right)$, and hence $K_{O}$, is $\mathbb{Z}$-acyclic. Moreover, it was conjectured in [14] that $\mathcal{N}\left(K_{O}\right)$ is homeomorphic to $Q$. Since $N_{O}$ is simply a triangulation of $Q$, this is indeed the case.

Let $A_{5}$ be the alternating group of even permutations of the set $\{1,2,3,4,5\}$. Define the subgroups

$$
\begin{aligned}
U & :=\left\{g \in A_{5}: g \cdot 2=2\right\} \cong A_{4}, \\
V & :=N_{A_{5}}(\langle(1,2,3,4,5)\rangle) \cong D_{5}, \\
W & :=N_{A_{5}}(\langle(1,3,5)\rangle) \cong D_{3},
\end{aligned}
$$

where $N_{A_{5}}(H)$ denotes the normalizer in $A_{5}$ of a subgroup $H$ of $A_{5}$. The stabilizer $U$ of the point 2 is isomorphic to the alternating group $A_{4}$ and has 12 elements. The subgroups $V$ and $W$ are isomorphic to the dihedral groups $D_{5}$ and $D_{3}$ with 10 and 6 elements, respectively.

Oliver takes as vertex set $E$ for the simplicial complex $K_{O}$ the 60 elements of $A_{5}$ and lets $A_{5}$ act transitively on $E$ by left multiplication. He defines $K_{O}$ to be the simplicial complex that has the left cosets of $U, V$, and $W$ as its (orbits of) maximal faces:

$$
K_{O}:=\bigcup_{g \in A_{5}} 2^{g \cdot U} \cup \bigcup_{g \in A_{5}} 2^{g \cdot V} \cup \bigcup_{g \in A_{5}} 2^{g \cdot W} .
$$

Theorem 15 (Oliver, see [10]). The 11-dimensional simplicial complex $K_{O}$ is vertexhomogeneous and $\mathbb{Z}$-acyclic.

Proof. By construction, $K_{O}$ is 11-dimensional and vertex-homogeneous. To see that $K_{O}$ is $\mathbb{Z}$-acyclic, we compute the nerve $\mathcal{N}\left(K_{O}\right)$ of $K_{O}$. As maximal faces of the nerve we get 60 triangles. By a suitable labeling of the vertices, $\mathcal{N}\left(K_{O}\right)$ turns out to be the triangulation $N_{O}$ of the $\mathbb{Z}$-acyclic identified dodecahedron $Q$ (see Fig. 5). Thus $K_{O}$ is $\mathbb{Z}$-acyclic by the Nerve Theorem (Theorem 4).

Let us have a closer look at the construction of $K_{O}$. By definition, $K_{O}$ is made up of three orbits of maximal faces consisting of five 12-element sets, six 10-element sets, and ten 6-element sets corresponding to the left cosets of the subgroups $U, V$, and $W$, respectively. In particular, $K_{O}$ is not pure! The complex $K_{O}$ is taut, and so is its nerve $N_{O}$. Hence, if we want to reconstruct $K_{O}$ from $N_{O}$, we see by Fig. 5 that $N_{O}$ has 60 triangles, so $K_{O}$ has 60 corresponding vertices. Further, we observe that there are five vertices of $N_{O}$ which have twelve neighboring triangles each, namely the original vertices 1-5 of the identified dodecahedron. Thus the nerve operation applied to $N_{O}$ will produce a 12-tuple as a maximal face of $K_{O}=\mathcal{N}\left(N_{O}\right)$ for each of these vertices. Similarly, the six vertices $6-11$ of $N_{O}$ have ten neighboring triangles and thus yield six 10-tuples as facets of $K_{O}$. The ten vertices 12-21 finally give ten 6-element sets as facets of $K_{O}$. Thus, alternatively to Oliver's algebraic construction, all information on $K_{O}$ can be obtained geometrically via the nerve operation from the picture of $N_{O}$ in Fig. 5 . 


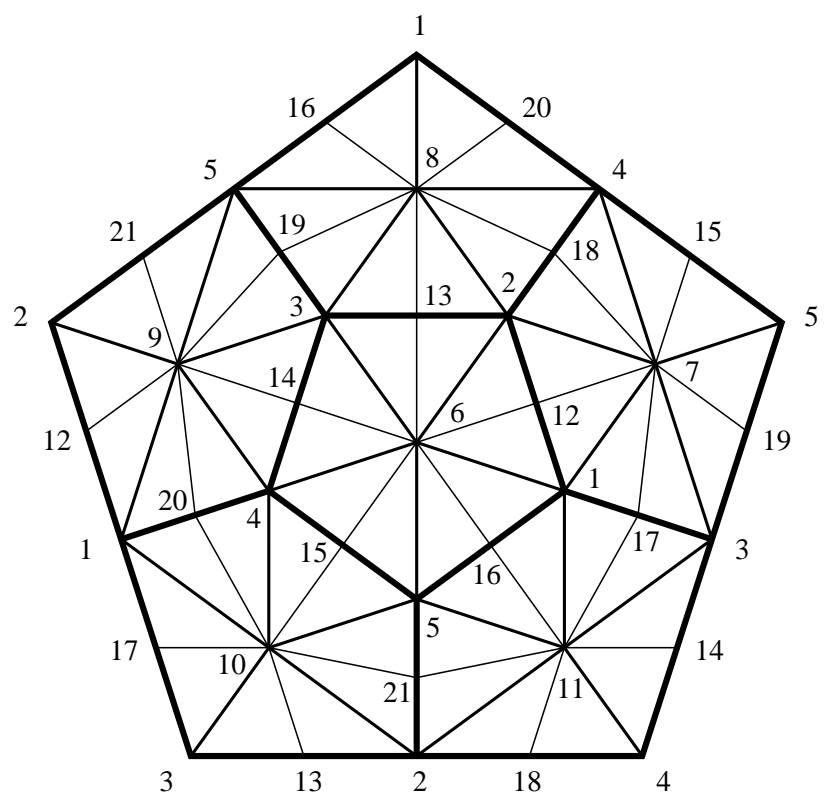

Fig. 5. Triangulation $N_{O}$ of the identified dodecahedron $Q$ with 60 triangles.

New $\mathbb{Z}$-Acyclic Vertex-Homogeneous Simplicial Complexes. In [11] we enumerated all vertex-homogeneous simplicial complexes with reduced Euler characteristic $\tilde{\chi}=0$ corresponding to a given group action on few vertices. For the $A_{5}$-action on 60 vertices it is hopeless to generate all vertex-homogeneous simplicial complexes with $\tilde{\chi}=0$ and then compute their homology in order to find $\mathbb{Z}$-acyclic examples. However, if we restrict our computer search to complexes that have only a few orbits of maximal faces with orbit size less than 30 , then, in particular, we obtain the above example $K_{O}$. Recall [11] that an $A_{5}$-orbit of $k$-sets on 60 vertices can have size less than 30 if and only if $\operatorname{gcd}(k, 60)>2$. We formed combinations of at most six orbits with at most two orbits of maximal faces of the same dimension. For every simplicial complex $K$ corresponding to one of these collections of orbits of facets, we computed the reduced Euler characteristic $\tilde{\chi}(\mathcal{N}(K))$ of the nerve complex of $K$. Whenever $\tilde{\chi}$ was zero, we computed the homology of $\mathcal{N}(K)$ with the program HOMOLOGY by Heckenbach [9]. Including $K_{O}$, we found five $\mathbb{Z}$-acyclic $A_{5}$-invariant complexes on 60 vertices that we denote by $K_{O}, K_{2}, K_{4}, K_{5}$, and $K_{6}$. The examples $K_{2}$ and $K_{4}$ are not taut, and it turns out that $K_{1}:=\mathcal{N}\left(\mathcal{N}\left(K_{2}\right)\right)$ and $K_{3}:=\mathcal{N}\left(\mathcal{N}\left(K_{4}\right)\right)$ are taut $A_{5}$-invariant $\mathbb{Z}$-acyclic simplicial complexes on 30 vertices. We believe that if we extended our search, then further complexes would appear.

Theorem 16. There are at least seven non-contractible $\mathbb{Z}$-acyclic simplicial complexes with a vertex-transitive $A_{5}$-action that are homotopy equivalent to the identified dodecahedron $Q$.

Table 1 gives an overview of the examples. All seven complexes can be characterized algebraicly, and this we do for $K_{1}$ to $K_{6}$ in the following. Moreover, we give geometric descriptions of the corresponding facet-homogeneous nerve complexes $N_{\mathrm{I}}$ to $N_{\mathrm{IV}}$. 
Table 1. $\mathbb{Z}$-acyclic vertex-homogeneous simplicial complexes with $A_{5}$-action.

\begin{tabular}{lcc}
\hline \multicolumn{1}{c}{ Complex } & Number of vertices & Dimension \\
\hline$K_{O}=\mathcal{N}\left(N_{O}\right)$ & 60 & 11 \\
$K_{1}=\mathcal{N}\left(N_{\mathrm{I}}\right)$ & 30 & 11 \\
$K_{2}=2 K_{1}$ & 60 & 23 \\
$K_{3}=\mathcal{N}\left(N_{\mathrm{II}}\right)$ & 30 & 5 \\
$K_{4}=2 K_{3}$ & 60 & 11 \\
$K_{5}=\mathcal{N}\left(N_{\mathrm{III}}\right)$ & 60 & 11 \\
$K_{6}=\mathcal{N}\left(N_{\mathrm{IV}}\right)$ & 60 & 11 \\
\hline
\end{tabular}

Remark. Although the examples $K_{O}$ and $K_{1}$ to $K_{6}$ are not contractible, by Proposition 11 there exist infinite series of contractible vertex-homogeneous simplicial complexes associated with $K_{O}$ and $K_{1}$ to $K_{6}$.

The $\mathbb{Z}$-Acyclic Complexes $K_{1}$ and $K_{2}$. Consider the subgroups of $A_{5}$,

$$
\begin{aligned}
& U:=\left\{g \in A_{5}: g \cdot 2=2\right\} \cong A_{4}, \\
& V:=N_{A_{5}}(\langle(1,2,3,4,5)\rangle) \cong D_{5} .
\end{aligned}
$$

Then the 24-element set

$$
A:=U \cup U \cdot(2,5,3)
$$

determines an $A_{5}$-orbit of size 5 . Define

$$
K_{2}:=\bigcup_{g \in A_{5}} 2^{g \cdot A} \cup \bigcup_{g \in A_{5}} 2^{g \cdot V}
$$

and

$$
K_{1}:=\mathcal{N}\left(\mathcal{N}\left(K_{2}\right)\right)
$$

Theorem 17. The examples $K_{1}$ and $K_{2}$ are $\mathbb{Z}$-acyclic vertex-homogeneous simplicial complexes on 30 and 60 vertices, respectively, with $K_{2}=2 K_{1}$.

Proof. The nerve complex $N_{\mathrm{I}}=\mathcal{N}\left(K_{1}\right)=\mathcal{N}\left(K_{2}\right)$ of $K_{1}$ and $K_{2}$ is the facethomogeneous triangulation of the identified dodecahedron $Q$ with 30 triangles (see Fig. 6).

As before in the case of $K_{O}$, we can easily reconstruct $K_{1}$ from the taut triangulation $N_{\mathrm{I}}$ of $Q$ as follows. $N_{\mathrm{I}}$ has thirty triangles and five vertices $1-5$ with twelve and six vertices 6-11 with five neighboring triangles. Thus, $K_{1}$ has thirty vertices and five 12-tuples and six 5-element sets as facets. 


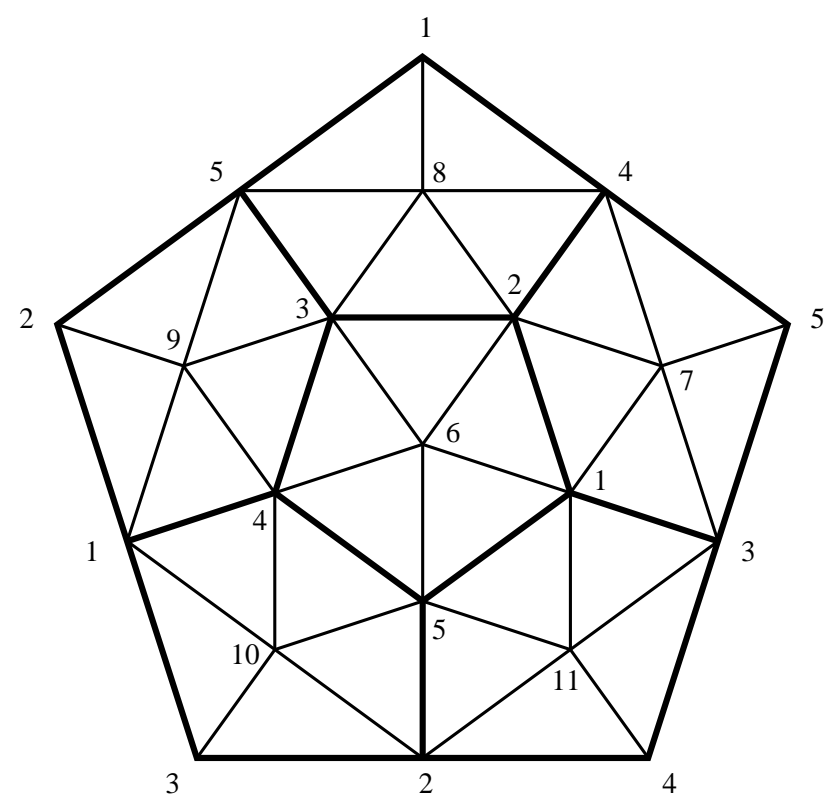

Fig. 6. Triangulation $N_{\mathrm{I}}$ of the identified dodecahedron $Q$ with 30 triangles

Since the group $A_{5}$ with 60 elements acts transitively on the 30 vertices of $K_{1}$, we can simply replace every vertex in each facet of $K_{1}$ by a pair of vertices to obtain a complex $K_{2}=2 K_{1}$. This complex with 60 vertices then has an obvious action by $A_{5}$.

The $\mathbb{Z}$-Acyclic Complexes $K_{3}$ and $K_{4}$. Take the subgroups of $A_{5}$,

$$
\begin{aligned}
U & :=\left\{g \in A_{5}: g \cdot 2=2\right\} \cong A_{4}, \\
V & :=N_{A_{5}}(\langle(1,2,3,4,5)\rangle) \cong D_{5}, \\
W & :=N_{A_{5}}\left(\langle(1,3,5)\rangle \cong D_{3},\right.
\end{aligned}
$$

and consider the 12-element set

$$
B:=W \cup W \cdot(3,4,5) .
$$

Define

$$
K_{4}:=\bigcup_{g \in A_{5}} 2^{g \cdot U} \cup \bigcup_{g \in A_{5}} 2^{g \cdot B} \cup \bigcup_{g \in A_{5}} 2^{g \cdot V},
$$

and set

$$
K_{3}:=\mathcal{N}\left(\mathcal{N}\left(K_{4}\right)\right) .
$$

The nerve $N_{\text {II }}=\mathcal{N}\left(K_{3}\right)=\mathcal{N}\left(K_{4}\right)$ of $K_{3}$ and $K_{4}$ is a taut three-dimensional facet-homogeneous simplicial complex with 30 tetrahedra (see Fig. 7). For every pentagon of $N_{O}, 5$ tetrahedra are glued in as indicated by the dashed lines. Since $N_{\text {II }}$ collapses to $N_{O}$, the complex $N_{\text {II }}$ is homotopy equivalent to $Q$. 


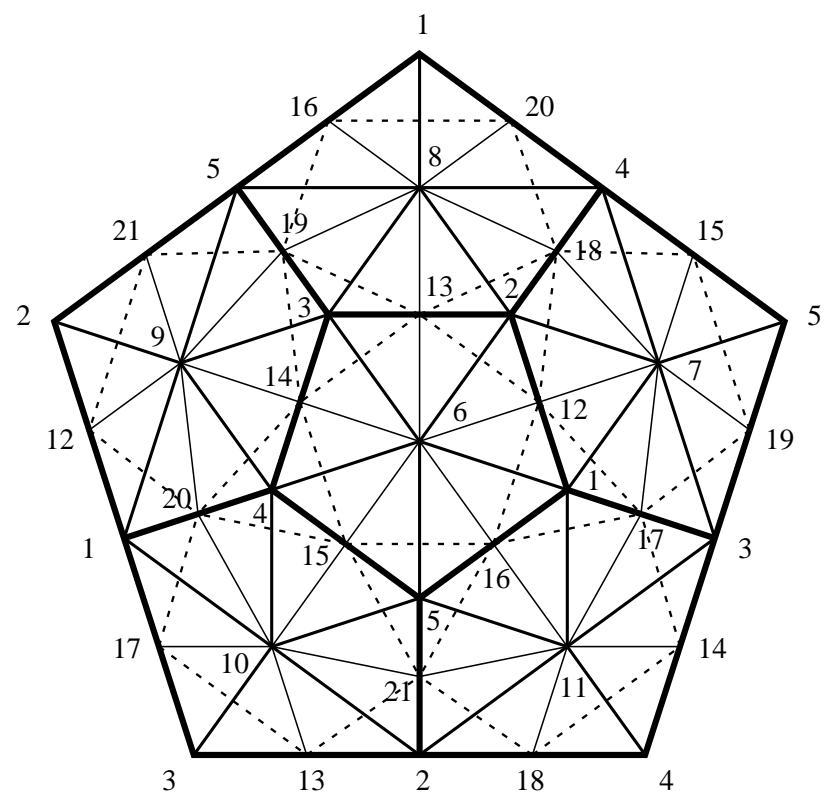

Fig. 7. Triangulation $N_{\text {II }}$ with 30 tetrahedra replacing the 60 triangles.

Theorem 18. The example $K_{3}$ provides a five-dimensional non-contractible $\mathbb{Z}$-acyclic vertex-homogeneous simplicial complex on 30 vertices.

We saw in [11] that there are no (non-trivial) two- and three-dimensional $\mathbb{Z}$-acyclic vertex-homogeneous simplicial complexes, and that if there were a four-dimensional example, then it would have $15,20,30$, or 60 vertices. Our attempts failed to find a four-dimensional example.

Conjecture 19. The complex $K_{3}$ with $f$-vector $f=(1,30,195,340,255,96,15)$ is the smallest example of a non-contractible $\mathbb{Z}$-acyclic vertex-homogeneous simplicial complex, with respect to dimension, the number of vertices, and the total number of faces. The join $K_{3} * K_{3}$ of dimension 11 with 60 vertices is, apart from a simplex, the smallest example of a contractible vertex-homogeneous simplicial complex.

The $\mathbb{Z}$-Acyclic Complexes $K_{5}$ and $K_{6}$. Let $U, V, W$, and $R$ be subgroups of $A_{5}$ with

$$
\begin{aligned}
U & :=\left\{g \in A_{5}: g \cdot 2=2\right\} \cong A_{4}, \\
V & :=N_{A_{5}}(\langle(1,2,3,4,5)\rangle) \cong D_{5}, \\
W & :=N_{A_{5}}(\langle(1,3,5)\rangle) \cong D_{3}, \\
R & :=\langle(1,2)(3,5),(1,3)(2,5)\rangle \cong \mathbb{Z}_{2} \times \mathbb{Z}_{2},
\end{aligned}
$$

and consider the 8-element set

$$
C:=R \cup R \cdot(2,3,4) .
$$




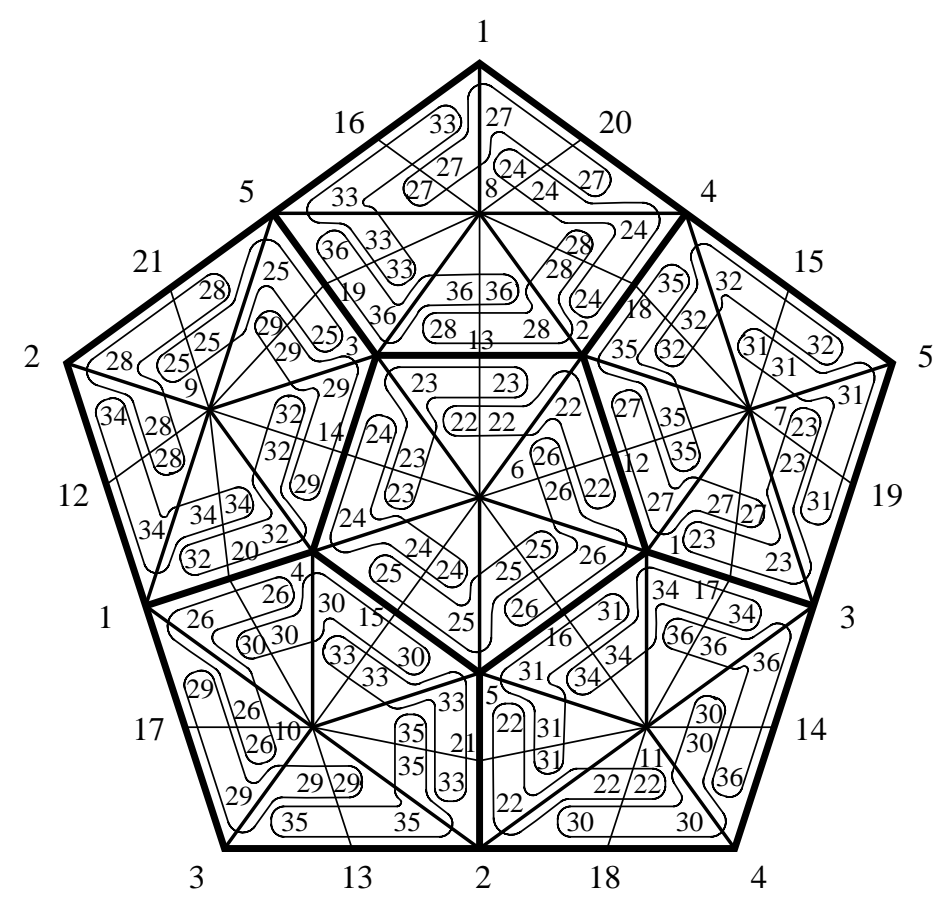

Fig. 8. Triangulation $N_{\text {III }}$ with 604 -simplices.

Define

$$
K_{5}:=\bigcup_{g \in A_{5}} 2^{g \cdot U} \cup \bigcup_{g \in A_{5}} 2^{g \cdot V} \cup \bigcup_{g \in A_{5}} 2^{g \cdot C} \cup \bigcup_{g \in A_{5}} 2^{g \cdot W}
$$

The nerve $N_{\mathrm{III}}=\mathcal{N}\left(K_{5}\right)$, composed of 604 -simplices on 36 vertices, is a taut facethomogeneous simplicial complex homotopy equivalent to $Q$. Figure 8 gives an illustration of $N_{\text {III }}$ via a projection onto $N_{O}$. To every one of the 60 triangles of the triangulation $N_{O}$ of $Q$ there uniquely corresponds a 4-simplex of $N_{\text {III }}$ which has as its vertices the three vertices of the respective triangle and in addition the two vertices that are placed within the triangle. For example, at the top of Fig. 8 we find the 4-simplices 18162733 and 18202427 . It can easily be verified that $N_{\text {III }}$ collapses to $N_{O}$, and thus $N_{\text {III }}$ is homotopy equivalent to $Q$. Each of the 15 vertices $22-36$ is contained in eight 4-simplices, hence these vertices contribute 158 -tuples as facets to $K_{5}=\mathcal{N}\left(N_{\mathrm{III}}\right)$.

Let once more $U, V, W$, and $S$ be subgroups of $A_{5}$ with

$$
\begin{aligned}
U & :=\left\{g \in A_{5}: g \cdot 2=2\right\} \cong A_{4}, \\
V & :=N_{A_{5}}(\langle(1,2,3,4,5)\rangle) \cong D_{5}, \\
W & :=N_{A_{5}}(\langle(1,3,5)\rangle) \cong D_{3}, \\
S & :=\langle(1,3)(4,5),(1,4)(3,5)\rangle \cong \mathbb{Z}_{2} \times \mathbb{Z}_{2},
\end{aligned}
$$




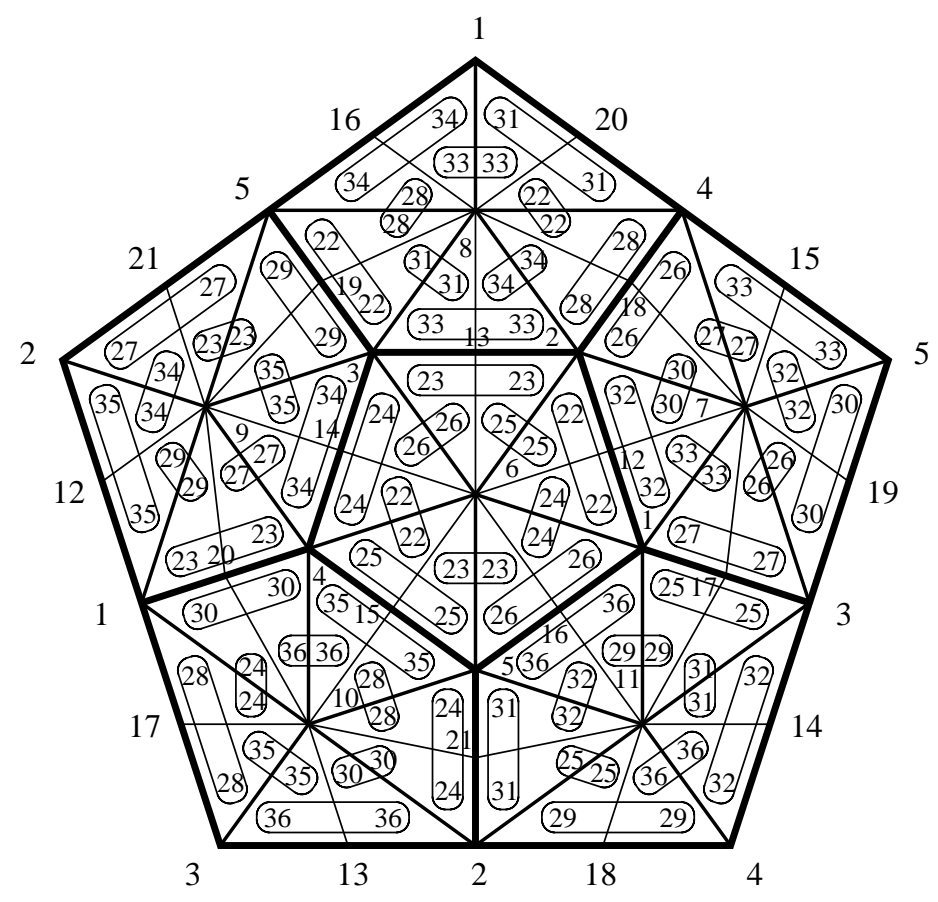

Fig. 9. Triangulation $N_{\mathrm{IV}}$ with 604 -simplices.

and consider the 8-element set

$$
D:=S \cup S \cdot(2,3,5) .
$$

Define

$$
K_{6}:=\bigcup_{g \in A_{5}} 2^{g \cdot U} \cup \bigcup_{g \in A_{5}} 2^{g \cdot V} \cup \bigcup_{g \in A_{5}} 2^{g \cdot D} \cup \bigcup_{g \in A_{5}} 2^{g \cdot W} .
$$

The nerve complex $N_{\mathrm{IV}}=\mathcal{N}\left(K_{6}\right)$ is again four-dimensional but combinatorially distinct from $N_{\text {III }}$, and provides another example of a taut facet-homogeneous simplicial complex homotopy equivalent to $Q$. The 604 -simplices of $N_{\text {IV }}$ are drawn in Fig. 9.

\section{Acknowledgments}

I thank Günter M. Ziegler for inspiring this work by bringing Oliver's example to my attention. Further, I am grateful to the referees for some helpful suggestions on the presentation of this paper.

\section{References}

1. M. Aigner and E. Triesch. Searching for an edge in a graph. J. Graph Theory 12, 45-57 (1988).

2. E. Babson, A. Björner, S. Linusson, J. Shareshian, and V. Welker. Complexes of not $i$-connected graphs. Topology 38, 271-299 (1999). 
3. A. Björner. Topological methods. In Handbook of Combinatorics (R. Graham, M. Grötschel, and L. Lovász, eds.), pp. 1819-1872. Elsevier, Amsterdam, 1995.

4. A. Björner and F. H. Lutz. Simplicial manifolds, bistellar flips and a 16-vertex triangulation of the Poincaré homology 3-sphere. Experimental Math. 9, 275-289 (2000).

5. B. Bollobás. Extremal Graph Theory. London Mathematical Society Monographs 11. Academic Press, London, 1978.

6. G. E. Bredon. Introduction to Compact Transformation Groups. Pure and Applied Mathematics 46. Academic Press, New York, 1972.

7. E. E. Floyd and R. W. Richardson. An action of a finite group on an $n$-cell without stationary points. Bull. Amer. Math. Soc. 65, 73-76 (1959)

8. B. Grünbaum. Nerves of simplicial complexes. Aequationes Math. 4, 63-73 (1970).

9. F. Heckenbach. Die Möbiusfunktion und Homologien auf partiell geordneten Mengen. Thesis for Diploma at University Erlangen-Nuremberg, 1997. (Computer program HOMOLOGY, http://www.mi.uni-erlangen.de/ heckenb/)

10. J. Kahn, M. Saks, and D. Sturtevant. A topological approach to evasiveness. Combinatorica 4, 297-306 (1984)

11. F. H. Lutz. Some results related to the Evasiveness Conjecture. J. Combin. Theory Ser. B 81, 110-124 (2001).

12. R. Oliver. Fixed-point sets of group actions on finite acyclic complexes. Comment. Math. Helv. 50, 155-177 (1975).

13. R. L. Rivest and J. Vuillemin. On recognizing graph properties from adjacency matrices. Theoret. Comput. Sci. 3, 371-384 (1976).

14. Y. Segev. Group actions on finite acyclic simplicial complexes. Israel J. Math. 82, 381-394 (1993).

15. P. A. Smith. Fixed points of periodic transformations. In Algebraic Topology (S. Lefschetz, ed.). American Mathematical Society Colloquium Publications 27, pp. 350-373. American Mathematical Society, New York, 1942

16. W. Threlfall and H. Seifert. Topologische Untersuchung der Diskontinuitätsbereiche endlicher Bewegungsgruppen des dreidimensionalen sphärischen Raumes. Math. Ann. 104, 1-70 (1931).

17. C. Weber and H. Seifert. Die beiden Dodekaederräume. Math. Z. 37, 237-253 (1933).

18. V. Welker. Constructions preserving evasiveness and collapsibility. Discrete Math. 207, 243-255 (1999).

19. A. C.-C. Yao. Monotone bipartite graph properties are evasive. SIAM J. Comput. 17, 517-520 (1988).

Received November 2, 2000, and in revised form July 2, 2001. Online publication November 7, 2001. 